Artigo / Article

\title{
Fatores de crescimento hemopoéticos nas síndromes mielodisplásicas
}

\section{Hematopoietic growth factors in myelodysplastic syndromes}

Elvira R. P. Velloso

\section{Introdução}

Os fatores de crescimento mais utilizados nas SMD são a eritropoetina (EPO) e o fator estimulante de colônias granulocíticas (G-CSF). Este último, além de ter sido testado em portadores de neutropenia como droga isolada, tem sido utilizado para potencializar a ação da EPO. Outros fatores de crescimento hemopoéticos foram investigados, mas os resultados preliminares com IL3 e da IL-6 em SMD se mostraram desanimadores, apresentando essas drogas efeitos colaterais importantes, como diminuição de plaquetas (IL-3) e diminuição de hemoglobina (IL-6). Também não está comprovada a eficácia da IL-11, para aumento da contagem de plaquetas em portadores de mielodisplasia., ${ }^{78}$

Um fluxograma para uso dos fatores de crescimento e de outras drogas utilizadas nas SMD de baixo risco é apresentado ao final do próximo capítulo. Também nesse mesmo local encontra-se descrita a proposta revisada da avaliação da eficácia ao tratamento nas SMD, segundo o IWG (International Working Group). ${ }^{6}$

\section{Uso da EPO}

Racional: promover o crescimento e diferenciação dos progenitores eritróides e inibir a apoptose, com doses farmacológicas. A elevação da taxa de hemoglobina e a redução da necessidade transfusional levariam à melhora da qualidade de vida dos pacientes responsivos a EPO.

\section{Resultado dos trabalhos do uso da EPO em SMD $^{1,3,10}$}

Vários esquemas utilizando eritropoetina em doses altas e variáveis, por via endovenosa ou subcutânea, foram empregados. Vários critérios de inclusão dos pacientes, com diferentes subtipos de SMD, e diferentes critérios de resposta devem ser levados em conta na análise dos dados.

Dois trabalhos de metanálise publicados em 1994 e 1995 mostraram melhora da anemia em $23,5 \%$ e $16,1 \%$ dos casos, respectivamente. O primeiro analisou dez trabalhos compreendendo 115 pacientes, mostrando melhor resposta para portadores de AREB. No segundo, a análise de 205 pacientes de 17 estudos indicou como fatores preditivos de melhor resposta o subtipo não ARSA, a dosagem sérica basal de EPO (s-EPO) < 200 U/l e a baixa necessidade transfusional.

Dois estudos randomizados avaliando a eficácia da EPO (EPO x placebo) foram publicados. O primeiro traba-

Serviço de Hematologia e Hemoterapia do Hospital das Clínicas da Universidade de São Paulo

Correspondência: Elvira Rodrigues Pereira Velloso

Rua Ministro Ferreira Alves, 1031, apto 183 A

05009-060 - São Paulo-SP

Telefones: (011) 3061-5544 r:312; (011) 9990-3279; Fax: (011) 3061-5544 - E-mail: elvira@sti.com.br 
lho, de 1991, randomizou vinte pacientes com AR ou ARSA, utilizando dose semanal de 1.600 ou $3.200 \mathrm{U} / \mathrm{kg}$ EV, com resposta em 12,5\% (1/8) pacientes. Não foi relatada toxicidade da droga. O grupo italiano analisou 87 pacientes com SMD de baixo risco $(\mathrm{Hb}<9 \mathrm{~g} / \mathrm{dl}$ e porcentagem de blastos na medula inferior a $10 \%$ ) na dose de $150 \mathrm{U} / \mathrm{kg} / \mathrm{d}$ SC por oito semanas, mostrando taxa de resposta de $36,8 \%$ no grupo tratado, versus $10,8 \%$ no grupo não tratado $(\mathrm{p}=0,007)$. Observou-se que a resposta era melhor para o subtipo AR, pacientes sem necessidade transfusional e s-EPO $<200$ U/l.

Em estudo de 1995, utilizando casuística expressiva, foram analisados 116 pacientes (84 AR/ARSA), sendo que cem deles completaram terapêutica igual ou superior a quatro semanas. A dose de EPO utilizada foi de $150 \mathrm{U} / \mathrm{kg} 3 \mathrm{x} /$ semana escalonada até $300 \mathrm{U} / \mathrm{kg} 3 \mathrm{x}$ semana. A taxa de resposta (aumento do hematócrito a 6\% do basal ou redução maior ou igual a $50 \%$ do inicial) foi de $28 \%$. A melhor resposta foi observada em AR e s-EPO $<100 \mathrm{U} / \mathrm{l}$, a melhor dose para reposta foi estimada em 150-200 U/kg 3x semana. Não houve relato de trombose, convulsão ou hipertensão.

No HC-FMUSP, de 03/94 a 07/96, 15 pacientes com SMD (cinco AR, seis ARSA, quatro AREB) receberam EPO na dose de $20.000 \mathrm{U} 2 \mathrm{x}$ semana. A s-EPO variou entre 28$3.180 \mathrm{U} / \mathrm{l}(\mathrm{nl} 17,7 \pm 7,5$ ), sendo abaixo de $100 \mathrm{U} / \mathrm{l}$ em quatro pacientes. Resposta completa (ausência de necessidade transfusional) foi observada em dois (13\%) pacientes, sendo os dois portadores de ARSA e com s-EPO abaixo de 100 U/l. ${ }^{11}$

Dois trabalhos de consenso em SMD analisaram o papel da EPO nas SMD: o do grupo italiano ${ }^{1}$ e o do grupo do Reino Unido. ${ }^{3} \mathrm{O}$ grupo italiano sugere o uso de EPO para pacientes com $\mathrm{Hb}$ abaixo de $10 \mathrm{~g} / \mathrm{dl}$, AR/ARSA e EPO sérica abaixo de $200 \mathrm{mU} / \mathrm{ml}$ (nível de evidência A), com doses superiores a 30.000 U/semana. O grupo do Reino Unido considera o uso de EPO na SMD como nível de evidência $\mathrm{A} / \mathrm{B}$.

\section{Uso de EPO associada a G-CSF ${ }^{1,3,9,10}$}

Os primeiros trabalhos da associação de EPO e GCSF foram publicados em 1993, mostrando melhora da anemia em aproximadamente $40 \%$ dos pacientes. Os fatores preditivos de resposta incluíram baixo nível de s-EPO, presença de sideroblastos em anel e pancitopenia menos avançada. Em mais de $90 \%$ dos casos foi observada elevação dos neutrófilos.

O grupo nórdico vem explorando essa associação de drogas nas SMD. Em trabalho analisando 87 pacientes (30 com AR, 31 ARSA, 32 com AREB e 5 com AREBt), utilizando doses escalonadas de G-CSF (0,3-3,0 $\mu \mathrm{g} / \mathrm{kg} / \mathrm{d} \mathrm{SC})$ e EPO (60-300 U/kg/d) por dez semanas, foi mostrada taxa de reposta eritróide de $36 \%$. Dois fatores se associaram à resposta: nível basal de s-EPO (100, 100-500 e $\geq 500$ U/l) e a necessidade transfusional de hemácias ( $<2$ ou $\geq 2$ unidades por mês). Utilizando-se essas variáveis era possível cal- cular a chance de resposta: alta probabilidade (74\%), média (23\%) e baixa (7\%). Estudo conduzido pelo mesmo grupo, utilizando doses escalonadas de G-CSF (30-75-150 $\mu \mathrm{g} /$ dia) e de EPO (5.000-10.000 U/dia) mostrou taxa de resposta de $38 \%$, sendo a mediana de sobrevida de 26 meses e a mediana de duração de resposta de 24 meses. Este modelo de resposta foi validado em trabalho prospectivo. Pacientes com EPO sérica abaixo de 500 U/l e necessidade transfusional menos de duas unidades por mês tiveram $61 \%$ de resposta enquanto pacientes com um dos critérios (EPO acima de $500 \mathrm{U} / \mathrm{l}$ ou necessidade transfusional maior ou igual a 2 U/mês) tinham 14\% de resposta. A mediana da duração da resposta foi de 23 meses, havendo melhora da qualidade de vida nos pacientes que apresentavam resposta. Em 2005 foram publicados os resultados a longo prazo do uso do esquema combinado, mostrando que pacientes com alta ou intermediária probabilidade de resposta e com IPSS de risco baixo ou intermediário 1 apresentavam respostas freqüentes e duradouras, enquanto outros pacientes não seriam candidatos a esse tratamento. ${ }^{9}$

Doses escaladas de G-CSF (1,5 $\mu \mathrm{g} / \mathrm{kg} /$ dia com ajuste para neutrófilos de 3.000 a 10.000) e de EPO (200 a 400 U/kg 3 vezes na semana) foram capazes de promover $61 \%$ de resposta eritróide a 12 semanas, chegando a $80 \%$ em 36 semanas. Poucos efeitos colaterais foram descritos, particularmente dor óssea, que em alguns casos pode ser limitante.

No serviço de hematologia do HC-FMUSP, entre 01/98 e 11/2001, 22 pacientes com SMD e anemia foram submetidos a protocolo de acordo com o nível sérico de EPO. Se s-EPO < 200 U/l recebiam EPO 20.000 U SC 2x semana e se EPO entre 200 e $500 \mathrm{U} / \mathrm{l}$ ou EPO $<200 \mathrm{U} / \mathrm{l}$ e falha terapêutica a EPO recebiam EPO 20.000 U 2x semana e G-CSF $300 \mu \mathrm{g} 2 \mathrm{x}$ semana. Resposta a EPO foi observada em 6/14 (42,8\%) pacientes, sendo os respondedores portadores de cariótipo normal e IPSS de risco baixo ou intermediário 1 e 5/6 com EPO < 100 U/l. Resposta a EPO+GCSF foi observada em $4 / 10(40 \%){ }^{2}$

O único trabalho randomizado (tratamento de suporte $x$ EPO+ G-CSF) avaliando efeitos na qualidade de vida, econômicos e de resposta foi publicado em 2004. Sessenta pacientes portadores de SMD com anemia leve e EPO basal inferior a $500 \mathrm{U} / 1$ foram randomizados. Apesar da taxa de resposta eritróide ser maior no grupo tratado (10/24 x 0/26, $\mathrm{p}=0,01$ ), não houve melhora na qualidade de vida medida por uma escala funcional (FACT-An). O tratamento com EPO+G-CSF foi bem mais caro do que o de suporte (26.723 euros versus 8.746 euros por paciente). ${ }^{4}$ Este trabalho foi questionado por alguns estudiosos, em relação à avaliação da qualidade de vida e por não responder a dúvida de ser a associação melhor do que o uso isolado de EPO.

Os dois trabalhos de consenso em SMD publicados antes deste último trabalho randomizado divergem em relação ao uso da associação de EPO e G-CSF nas SMD. O grupo italiano ${ }^{1}$ não recomenda a associação de G-CSF, dado 
não haver evidências de melhor resposta em relação a EPO isolada. $\mathrm{O}$ grupo do Reino Unido ${ }^{3}$ acredita haver evidências para uso e sugere que nas AR e AREB com anemia, necessidade transfusional $<2$ U/mês e s-EPO $<200$ U/l seja utilizada EPO na dose de 10.000 U/dia x 6 semanas e não havendo resposta dobrar a dose de EPO ou associar G-CSF, na dose de 75-150-300 $\mu \mathrm{g} / \mathrm{semana}$, para manter leucócitos entre 6.000 e $10.000 / \mathrm{mm}^{3}$ e a seguir ajustar a dose. Para portadores de ARSA, anemia, s-EPO $<500 \mathrm{U} / \mathrm{l}$, $\mathrm{tx}<2 \mathrm{U} /$ mês sugere o uso da associação de EPO e G-CSF nas doses já citadas.

\section{Uso de G-CSF e GM-CSF ${ }^{1,3,5}$}

O uso de fatores de crescimento para granulócitos e para granulócitos e monócitos (G-CSF/ GM-CSF), apesar de promover aumento na contagem dos neutrófilos em $60 \%$ 100\% dos casos, tem eficácia discutível nas SMD.

Em estudo randomizado, utilizando-se GM-CSF x observação em pacientes com SMD, viu-se que, apesar de ocorrer aumento de neutrófilos, a taxa de infecção não se alterava. Observou-se também que no grupo tratado não havia aumento da $\mathrm{Hb}$, havia redução da contagem de plaquetas e não havia diferença quanto à taxa de evolução para LMA. Também em trabalho randomizado comparando diferentes doses de GM-CSF, realizado pelo EORTC (European Organization for Research and Treatment of Cancer), 44 (66\%) dos 82 pacientes apresentaram resposta granulocítica, havendo maior resposta em portadores de cariótipo normal. Em um estudo, pacientes submetidos a altas doses de GMCSF apresentaram menor taxa de infecções.

Boa tolerabilidade, elevação de neutrófilos e queda no número de blastos na $\mathrm{MO}$ em alguns casos foram observadas em pacientes submetidos ao uso de G-CSF por 7 a 14 dias. A utilização da droga por períodos mais prolongados (meses) levou a redução no número de infecções quando o número absoluto de neutrófilos estava acima de $1.500 / \mathrm{mm}^{3}$, em um estudo. Um estudo randomizado mostrou que o grupo tratado com G-CSF mostrava aumento de neutrófilos, sem alteração da taxa de evolução para LMA, entretanto plaquetopenia era um efeito colateral importante.

Efeitos colaterais do tipo Síndrome flu-like e síndrome do extravasamento capilar são mais observados com GMCSF do que com G-CSF.

Baseado nestes dados, o uso do G-CSF/GM-CSF não está indicado para melhora da condição hematológica em portadores de SMD. O uso desses fatores deve ser individualizado em pacientes neutropênicos em vigência de infecções, e profilaticamente para pacientes com graves neutropenias e infecções, para manter contagem de neutrófilos acima de $1.000 / \mathrm{mm}^{3}$.

\section{Abstract}

Treatment with growth factors, particularly erythropoietin (EPO) alone or in association with granulocyte colony-stimulating factor (G-CSF), has been useful in the management of anemia in lowrisk patients with myelodysplastic syndromes (refractory anemia, refractory anemia with ringed sideroblasts, low or intermediate-1 IPSS groups). Two pre-treatment variables (serum EPO levels and red blood cell-transfusion needs) can predict erythroid responses to these high cost treatments. The indiscriminate use of G-CSF in neutropenic patients is not recommended although it can be useful in specific situations. Rev. bras. hematol. hemoter. 2006;28 (3): 210-212.

Key words: Myelodysplastic syndromes; anemia; erythropoietin; colony-stimulating factor; G-CSF.

\section{Referências Bibliográficas}

1. Alessandrino EP, Amadori S, Barosi G, Cazzola M, Grossi A, Liberato LN, et al; Italian Society of Hematology. Evidence- and consensus-based practice guidelines for the therapy of primary myelodysplastic syndromes. A statement from the Italian Society of Hematology. Haematologica 2002; 87 (12):1286-306.

2. Belisário Filho V, Velloso ERP, Pereira J, Beitler B, Dorlhiac-LLacer PE, Chamone DAF. Uso de EPO associado ou não a G-CSF em SMD. Anais do $25^{\circ}$ Congresso Brasileiro de Hematologia e Hemoterapia, pg 127, 2002.

3. Bowen D, Culligan D, Jowitt S, Kelsey S, Mufti G, Oscier D, et al; UK MDS Guidelines Group. Guidelines for the diagnosis and therapy of adult myelodysplastic syndromes. Br J Haematol 2003;120(2):187-200.

4. Casadevall N, Durieux P, Dubois S, Hemery F, Lepage E, Quarre MC, et al. Health, economic, and quality-of-life effects of erythropoietin and granulocyte colony-stimulating factor for the treatment of myelodysplastic syndromes: a randomized, controlled trial. Blood 2004;104(2):321-7.

5. Cazzola M, Anderson JE, Ganser A, Hellstrom-Lindberg E, Cazzola M, Anderson JE, et al. A patient-oriented approach to treatment of myelodysplastic syndromes. Haematologica 1998;83(10):910-35.

6. Cheson BD, Greenberg PL, Bennett JM, Lowenberg B, Wijermans PW, Nimer SD, et al. Clinical application and proposal for modification of the International Working Group (IWG) response criteria in myelodysplasia. Blood 2006;108(2):419-25 .

7. Gordon MS. Advances in supportive care of myelodysplastic syndromes. Semin Hematol 1999;36(4 Suppl 6):21-4.

8. Heaney ML, Golde DW. Myelodysplasia. N Engl J Med 1999;340 (21):1649-60.

9. Hellstrom-Lindberg E. Update on supportive care and new therapies: Immunomodulatory drugs, growth factors and epigenetic-acting agents. In "Hematology 2005. American Society of Hematology Education Program Book", 2005. p.161-6.

10. Steensma DP, Bennett JM. The myelodysplastic syndromes: diagnosis and treatment. Mayo Clin Proc 2006; 81(1):104-130.

11. Velloso ERP, Mota A, Gualandro S, Melo N, Beitler B, Dorlhiac-Llacer PE, et al. Uso de eritropoetina em síndromes mielodisplásicas. Boletim da Sociedade Brasileira de Hematologia e Hemoterapia, XVIII (supl):44P, 1996.

Avaliação: Editor e dois revisores externos.

Conflito de interesse: não declarado

Recebido: 14/03/2006

Aceito após modificações: 11/09/2006 